\title{
Validación de un Cuestionario para Evaluar Riesgo de Caries en Preescolares en Santiago, Chile
}

\author{
Validation of a Questionnaire to Assess Risk of Caries \\ in Preschool-Age Children in Santiago, Chile
}

Y. Candiales¹; R. Cabello; G. Rodríguez; L. Marró3; I. Morales²; B. Urzúa² \& J. Sánchez

CANDIALES, Y. ; CABELLO, R.; RODRÍGUEZ, G. ; MARRÓ, L. ; MORALES, I.; URZÚA, B. \& SÁNCHEZ, J. Validación de un cuestionario para evaluar riesgo de caries en preescolares en Santiago, Chile. Int. J. Odontostomat., 12(1):135-155, 2018.

RESUMEN: La Caries Temprana de la Infancia (CTI) es una forma de caries dental agresiva que afecta a niños, y en el último reporte nacional reveló una prevalencia de caries del $50 \%$ en niños de 4 años de edad de la Región Metropolitana de Santiago (Soto et al., 2007). El objetivo de este estudio es validar un cuestionario que permita recolectar información relacionada con diversos factores de riesgo de caries en niños preescolares. Para la validación del cuestionario se determinó la validez de convergencia y discriminación, la consistencia interna y la confiabilidad test retest del instrumento en dos muestras independientes. Se aplicó el instrumento al cuidador principal de 118 preescolares entre 24 a 71 meses de edad, que asisten a jardines infantiles de dependencia particular (bajo riesgo de caries) y la Fundación INTEGRA (alto riesgo de caries) en la Región Metropolitana de Santiago, Chile. Se realizaron exámenes clínicos dentales por 2 odontólogos calibrados utilizando en el criterio OMS e ICDAS II. Se estimó un modelo de regresión logística y se evalúo la capacidad de discriminación del puntaje a través de una curva ROC. El cuestionario mostró una validez de discriminación de 0,95 entre ambos grupos y de la pregunta global 0,61 y una consistencia interna del cuestionario de 0,72. En la validez de convergencia se encontró que no existe asociación estadísticamente significativa entre el puntaje del cuestionario y la pregunta global dicotomizada (OD) 1,061. No obstante, se evidenció que si existe asociación estadísticamente significativa entre el puntaje del cuestionario y el grupo riesgo de caries (bajo y alto) (OD) 1,961. La estabilidad temporal mediante comparaciones Test - Retest calculado con el índice de Kappa osciló entre 0,37 a 1. Por lo tanto, se puede concluir que el presente cuestionario es un instrumento válido para discriminar riesgo de caries, permitiendo un mejor análisis de los determinantes de la caries dental en la población preescolar chilena.

PALABRAS CLAVE: dental caries, evaluación de riesgo, preescolares.

\section{INTRODUCCIÓN}

La caries dental es una enfermedad de carácter crónico y de etiología multifactorial que se inicia en la infancia temprana, en ausencia de tratamiento progresa hasta la destrucción de los dientes (Edelstein, 2006; Baelum et al., 2007). El 60 a $90 \%$ de los escolares a nivel mundial presenta lesiones de caries con cavitación evidente, según la Organización Mundial de la Salud (Petersen, 2003).

Conseguir una salud oral adecuada generalmente requiere de un alto costo económico, lo que limita los recursos y la posibilidad de tener fácil acceso a una oportuna atención odontológica. El sistema de salud en Chile tiene una capacidad limitada para proveer una atención dental temprana, especialmente a la comunidad preescolar (Petersen).

La severidad de la caries dental aumenta con la edad entre los 2 a 5 años. A los 2 años se reporta un coed (dientes temporales, cariados obturados y extraídos) de 0,54 $\pm 1,53$; a los 4 años de $2,32 \pm 3,27$ y a los 6 años un coed de 3,7 y un COPD de 0,15 (Ceballos et

${ }^{1}$ Área de Cariología, Departamento de Odontología restauradora, Facultad de Odontología, Universidad de Chile, Sergio Roberto Livingstone Polhammer 943, Independencia, Región Metropolitana, Chile. CL 7610658

2 Instituto de Ciencias Odontológicas, Facultad de Odontología, Universidad de Chile, Sergio Roberto Livingstone Polhammer 943 , Independencia, Región Metropolitana, Chile. CL 7610658

${ }^{3}$ Área de Cariología, Departamento de Odontología restauradora, Facultad de Odontología, Universidad de los Andes, Monseñor Álvaro del Portillo número 12455, Región Metropolitana, Chile. CL 7591538 
al., 2007; Soto et al.). Estas cifras indican que en un periodo de 4 años, cada niño desarrolla al menos 3 nuevas lesiones de caries en su boca (Ceballos et al.).

La evaluación del riesgo es un componente esencial en el proceso de toma de decisión para la prevención y el manejo de la caries dental. Debido a su carácter multifactorial, se debe recopilar información relacionada con el entorno individual, social y comunitario del preescolar. Para ello, se han desarrollado una amplia variedad de cuestionarios que abordan aspectos biológicos, sociales, conductuales, culturales, relacionados con el desarrollo de la enfermedad (Ramos-Gómez et al., 2002; Marshall et al., 2003; Finlayson et al., 2006; Patir et al., 2008; Gao et al., 2010; MacRitchie et al., 2012).

Un cuestionario es un instrumento diseñado para poder cuantificar y universalizar la información recogida por medio de él y estandarizar el procedimiento de entrevista. Su finalidad es conseguir la comparabilidad de los datos recogidos. Por esta razón, previo a su aplicación a una población objetivo, debe ser sometido a un proceso de validación sistemática que asegure su fiabilidad (Ramos-Gómez et al.; Marshall et al.). Sin embargo, los cuestionarios reportados en la literatura (Ramos-Gómez et al.; Kalwitzki et al., 2002; Marshall et al.; Finlayson et al.; Patir et al.; Gao et al.; MacRitchie et al.) aplicados para evaluar riesgo de caries en preescolares no han sido sistemáticamente validados en este grupo etario, dificultando la comparación de diferentes estudios y la interpretación de sus resultados.

Por estos motivos, se creó un "Cuestionario para evaluar riesgo de Caries en preescolares chilenos" y el propósito de este trabajo es determinar la validez de discriminación y fiabilidad de este cuestionario, con la finalidad de contar con un instrumento confiable que permita recolectar información relacionada con las determinantes de la caries dental en la población preescolar chilena vulnerable.

\section{MATERIAL Y MÉTODO}

El instrumento: El cuestionario fue creado por un grupo de investigadores de las áreas de Cariología, Salud Pública, Ciencias del Comportamiento y Bioquímica de la Facultad de Odontología de la Universidad de Chile, que realizó una revisión bibliográfica de la literatura relacionada con las determinan- tes de riesgo de caries en preescolares. A este instrumento se le determinó la validez de contenido, pretest cognitivo y se ponderó la puntuación de los dominios e ítems. La versión preliminar fue sometida a análisis por un panel de 4 expertos con el objetivo de evaluar la comprensión lingüística y la ponderación sugerida para cada ámbito y cada pregunta del cuestionario. Los expertos fueron seleccionados considerando su experiencia profesional e investigativa en el ejercicio de la odontología. Se incluyeron profesionales considerados "expertos" en Cariología, Odontología Restauradora y Metodología de la investigación. Este cuestionario modificado se aplicó a un piloto de 30 cuidadores principales de preescolares de 24 a 71 meses de edad, para realizar el pre-test cognitivo. Finalmente el cuestionario comprendió 7 dominios con un total de 108 preguntas (Formulario). Los dominios a evaluar se describen a continuación:

-Dominio I Sociodemográfico (14 preguntas).

-Dominio II Antecedentes de salud oral del preescolar y del cuidador (21 preguntas).

-Dominio III Construcción social de enfermedad: nociones y percepciones del sujeto de investigación (14 preguntas).

-Dominio IV Autopercepción de salud oral y de necesidad de tratamiento (6 preguntas).

-Dominio V Hábitos de alimentación (18 preguntas). -Dominio VI Hábitos de higiene oral (13 preguntas). -Dominio VII Exposición a microorganismos (22 preguntas).

Además, contiene un componente de identificación y contacto con un total de 16 preguntas sobre datos relacionados al nombre y sexo del niño(a), nombre del encuestado, dirección, correo electrónico, tipo de hogar y remuneración. Para evaluar la creencia del cuidador principal del niño (a) respecto de la condición de riesgo de caries de su hijo (a), se incorporó una preguntaglobal $(P G)$ al final del cuestionario.

Ponderación de los dominios, escalas de medición. Mediante un consenso realizado por 5 expertos en el área de: Cariología, Salud Pública, Restauradora, Odontopediatría y Odontología General; fue ponderado el cuestionario de identificación y contacto; dominio y pregunta de cada dominio (Tabla I).

Población y muestra. Fueron seleccionados 118 preescolares sanos (rango de edad de 24-71 meses, de ambos sexos), los cuales participaron voluntariamente de este estudio firmando el consentimiento informado correspondiente por su cuidador principal. 
CANDIALES, Y. ; CABELLO, R.; RODRÍGUEZ, G. ; MARRÓ, L. ; MORALES, I.; URZÚA, B. \& SÁNCHEZ, J. Validación de un cuestionario para evaluar riesgo de caries en preescolares en Santiago, Chile. Int. J. Odontostomat., 12(1):135-155, 2018.

Tabla I. Ponderación de los dominios, escalas de medición.

\begin{tabular}{llc}
\hline \multicolumn{1}{c}{ Dominio } & \multicolumn{1}{c}{ Nombre } & Ponderación \\
\hline I & Sociodemográfico & $13,6 \%$ \\
II & Antecedentes de salud bucal del preescolar y del cuidador & $12,6 \%$ \\
III & Construcción social de enfermedad & $11,7 \%$ \\
IV & Autopercepción de salud bucal y de necesidad de tratamiento & $9 \%$ \\
V & Hábitos de alimentación & $17 \%$ \\
VI & Hábitos de higiene oral & $18 \%$ \\
VII & Exposición a microorganismos & $4,5 \%$ \\
& Cuestionario de identificación y contacto & $13,6 \%$ \\
\hline
\end{tabular}

\section{ANEXO}

Cuestionario utilizado como instrumento de evalusción de factores de riesgo de caries dental.

Encuesta $\mathrm{N}$

Carnet de identidad del niño:

Fecha:

1 I

Dominio I: Sociodemográfico.

Este domino intenta rocoger información de valoración de la sìtusción socioeconómica del entorno del niñolniña.

1. ¿Es ustod la parsona que se encarga principalmente del cuidado del niñoiniña? Si__ No

Sí es "No" ¿Quién se encarga? (Contactar al cuidador principal y reiniciar la encuesta)

2. Nacionalidad de la madre: (Si es extronjera, pasar a la siguiente pregunta)

3. ANio on el que la madre llegó al pais: Nacionalidad del niño/niña:

4. ¿Cuál es su actividad labord principa? (No son excluyentes, marcar todas las que correspondan)

1) Dueña de caรล

2) Obrero u operario

3) Asesora del hogar

4) Oficinista o Junior

5) Estudiante 1) Básica incomplata

2) Básica completa

3) Media incompleta

4) Media completa

5) Técrico incomplata
6) Profesiona

7) Empresario

8) Ejecutivo

9) Comerciante

10) Transportista
11) Trabajo estacional (tempororos)

12) Trabajo agricola

13) Artista o artesan

14) Otra

15) No tiene actividad

6. ¿Qué sistema de previsión en salud bene el niño/niña?

FONASA

(Nivel) 
CANDIALES, Y. ; CABELLO, R.; RODRÍGUEZ, G. ; MARRÓ, L. ; MORALES, I.; URZÚA, B. \& SÁNCHEZ, J.Validación de un cuestionario para evaluar riesgo de caries en preescolares en Santiago, Chile. Int. J. Odontostomat., 12(1):135-155, 2018.

ISAPRE

OTRO

7. ¿En dónde realiza el control del niño sano?

1) Consulta privad

2) Consultorio (Centro de Salud Familiar)

3) Hospital ¿Cusl?

4) No realiza

8. ¿Dónde se atiende generalmente el niño/niña en caso de enfermedad?

1) Cansulta privada

3) Hospital

2) Consultorio (Centro de Salud Familar)

9. ¿Dónde se atiende generalmente el niño/niña por razones dentales?

1) Consulta privada

2) Consultorio (Centro de Salud Familar)

3) Haspital ¿Cuál?

4) No se atiende

10. ¿Qué sistema de previsión on salud tiene usted?

FONASA

Nivel

ISAPRE

OTRO

11. ¿Dónde se atiende generalmente usted por razones médicas?

1) Cansulta privada

2) Consultorio (Centro de Salud Familiar)

3) Hospìtal ¿Cuàl?

4) No se atiende

12. ¿Dónde se atiende generalmente usted por razones dentales?

1) Consulta privada

2) Consultorio (Centro de Salud Famiiar)

3) Hospìtal ¿Cuál?

4) No se atiende

13. ¿Cuántos adultos viven en la casa dande vive el niño/niña?

14. ¿Cuántos niños/niñas más víven en la casa donde vive el niño/niña?

\section{Dominio II: Antecedentes de salud bucal del niño y del cuidsdor.}

Este dominio recoge información sobre el historial de salud bucal del niño y del cuidador. 
CANDIALES, Y. ; CABELLO, R.; RODRÍGUEZ, G. ; MARRÓ, L. ; MORALES, I.; URZÚA, B. \& SÁNCHEZ, J. Validación de un cuestionario para evaluar riesgo de caries en preescolares en Santiago, Chile. Int. J. Odontostomat., 12(1):135-155, 2018.

\begin{tabular}{|c|c|c|}
\hline 15. ¿Nguna vez le ha revisado las dientes a su nirio/ niña en busca de caries (picaduras)? & Si & No \\
\hline 16. ¿Tiene el niño/niña alguna caries (picadurn) en este momento? & Si & No \\
\hline 17. ¿Ha tenido el niñainiña caries (picaduras) o tapoduras en el pasado? & Si & No \\
\hline 18. ¿Le han sacado dientes o muelas al nirioiniña debido a caries (picaduras)? & Si & No \\
\hline 19. ¿Los otros nincelnifnas que viven en la casa, han tenido caries (picaduras) o tapaduras)? & Sा & No \\
\hline 20. ¿Tiene el niño/niña problemas para masticar? & Si & No \\
\hline 21. ¿Tiene el niño/niña un diente o muela que le duele? & Si & No \\
\hline 22. ¿Piensa que al niñoiniña le gusta cómo se ven sus dientes (como lucen)? & Si & No \\
\hline 23. ¿Ha ido el nirio/niña alguna vez al den6sta? & Si & No \\
\hline 24. ¿Ha tenido usted alguna caries (picadura) en el posado, que ha sido restaursda/tapada? & Si & No \\
\hline 25. ¿En este momento, tiene alguna caries (picadura) que no esté restaurada/tapada? & Si & No \\
\hline 26. ¿A ustod, le malesta como se ven sus dientes? & Si & No \\
\hline 27. ¿Piensa que usted necesita tratamiento de caries (picaduras) en este momento? & Si & No \\
\hline 20. ¿Va usied regularmente al dentista? & Si & No \\
\hline 29. ¿Tiene usted miedo de ir al dentista? & Sा & No \\
\hline 30. ¿Usa usted pasta dental? & Si & No \\
\hline 31. ¿Usa usted otros productos de higiene con flíor para sus dientes (enjuague bucal, otro)? & Si & No \\
\hline 32. ¿Le han sacedo a usted, algunos de sus dientes ___ o muelas ___? & Si & No \\
\hline
\end{tabular}

33. ¿Cuál fue el peso y talla del niño/niña al nacer? Pesc

Tallar

34. ¿Por ouánto tiempo el niño/niña recibió pecho? Meses: 
CANDIALES, Y. ; CABELLO, R.; RODRÍGUEZ, G. ; MARRÓ, L. ; MORALES, I.; URZÚA, B. \& SÁNCHEZ, J. Validación de un cuestionario para evaluar riesgo de caries en preescolares en Santiago, Chile. Int. J. Odontostomat., 12(1):135-155, 2018.

35. ¿Ha estado el niño/niña en tratamiento prolongado con alguno de los siguientes remedias?

1) Para la alergia

3) Para la epilepsia

5) Para otra enfermedad
2) Para el asma

4) Para la hiperactividad

¿Cuál enfermedad?
6) Ninguna

\section{Dominio Ill: Construcción social de enfermedad.}

Este dominio descrbe las nociones y percepciones del sujeto de investigación, tal cual se expresan en su discurso. Alude a percepción de riesgo, etiología de la enfermedad e itinerario terapéufico. No debe inducirse respuestas. No son excluyentes en su mayoria. El encuestador soblo pregunta, pero no entrega las alternativas a los encuestadoe, pues, de lo contrario, no se recoge el dato espontáneo sino el dato ya procesado por el 'deber ser".

36. ¿Dónde adquirió conocimientee sobre las caries? (preguntas no excluyentes)

1. En un centro educacional básioo o secundario (escuela)

2. En la casa, o entre conocidoe

3. En Internet $u$ otros medias de comuricación y pronsa

4. En un centro de salud o a través de un profesional de la salud

5. En ninguna parte, no tengo conocimiento

6. Otro

37. ¿Porqué piensa que ha llegado a tener caries? (no excluyente)

1. No sabe

2. Por no ir al dentista freouentemente

3. Por besar o usar vasce, cepillos u otro objeto de una persana con caries

4. Porque tiene malos dientes

5. Porque sus podres tienen / tenlan malos dientes

6. Porque no se cepila las dientes con frecuencia

7. Por una mala alimentación o por comer duloes

8. Por descuido de loe padres o familiares

9. Otra

38. ¿Qué tendria que ver Ud. en el niño/niña, para llevarlo/a al dentista? 
CANDIALES, Y. ; CABELLO, R.; RODRÍGUEZ, G. ; MARRÓ, L. ; MORALES, I.; URZÚA, B. \& SÁNCHEZ, J. Validación de un cuestionario para evaluar riesgo de caries en preescolares en Santiago, Chile. Int. J. Odontostomat., 12(1):135-155, 2018.
1. Dolor
2. Olor
3. Dientes chuecos
4. Manchas on los dientes
5. Fractura de los dientes
6. Otro

39. De los motivas antes mencionados, establezce el orden de importancia, donde 1 es lo más importante.

\begin{tabular}{|l|l|}
\hline Nodel mobivo & Importancia \\
\hline & \\
\hline & \\
\hline & \\
\hline & \\
\hline
\end{tabular}

40. ¿Cuida Ud. que el niño/niña no tenga caries?

Si

No

41. ¿Por qué? (dependiendo de lo que conteste en progunta anterior)

Sies 'no' ¿Por qué no?

1) No tengo dinero para llevarlo al dentista [6 mi seguro de salud (fapsss o ispopes) no tiene cobertura]

2) Tiene buena dentadura

3) No sé cómo cuidarlo

4) Lo llevo al dentista sólo ouando tiene un problema. ¿Cuándo por ejemplo?

5) No necesito ouidarlo pues tenemos prácticas saludables (conduoe a la aliernativa 6)

6) Otro

Si es "si ¿Cómo lo cuida?

1) Se copilla o le cepilla los dientes

2) Lo llevo al dentista cada cierto tiempo sólo para que lo revisen 
CANDIALES, Y. ; CABELLO, R.; RODRÍGUEZ, G. ; MARRÓ, L. ; MORALES, I.; URZÚA, B. \& SÁNCHEZ, J.Validación de un cuestionario para evaluar riesgo de caries en preescolares en Santiago, Chile. Int. J. Odontostomat., 12(1):135-155, 2018.

3) No le doy alimentoe que le hagan mal para los dientes ¿Cus̉les?

4) Le day almentos que le hacen bien para los dientes. ¿Cuáles?

5) Utilizo algún producto especial para cuidarle los dientes ¿Cuál?

6) Otro

42. En general, cuando su niño tiene algún problema dental, ¿Dónde consulta?. Responda donde suele consultar primero, segundo y en tercer lugar. (Responder tal como refiere el sujeta; anotando el orden de las respuastas tal oual se da. No forzar tercera alternativa)

1) En la farmacia

2) En la famila, el barrio o con amigos

3) En internet u atro medio de comunicación

4) En centro de atención dental público

5) En un centro de atención dental privado

6) Medicina al:ernativa

7) Otro

\begin{tabular}{|c|c|c|c|c|}
\hline $\begin{array}{l}\text { ¿Qué tan de acuerdo está usied con las siguientes } \\
\text { afimaciones? }\end{array}$ & De acuendo & $\begin{array}{l}\text { Ni de acuardo ni } \\
\text { en desacuerdo } \\
(+0-)\end{array}$ & En & No sé \\
\hline $\begin{array}{l}\text { 43. La mayoria de los adultos perderán todos sus } \\
\text { dientes a medida que ellos envejecen. }\end{array}$ & & & & \\
\hline $\begin{array}{l}\text { 44. La mayoria de los niños tendrán caries } \\
\text { (picaduras). }\end{array}$ & & & & \\
\hline $\begin{array}{l}\text { 45. Los nifios necesitan flúor para cuidar sus } \\
\text { dientes y los adultos no. }\end{array}$ & & & & \\
\hline $\begin{array}{l}\text { 46. El tipo de comida y bebida que un nirio come o } \\
\text { toma puede causar caries (picaduras). }\end{array}$ & & & & \\
\hline
\end{tabular}




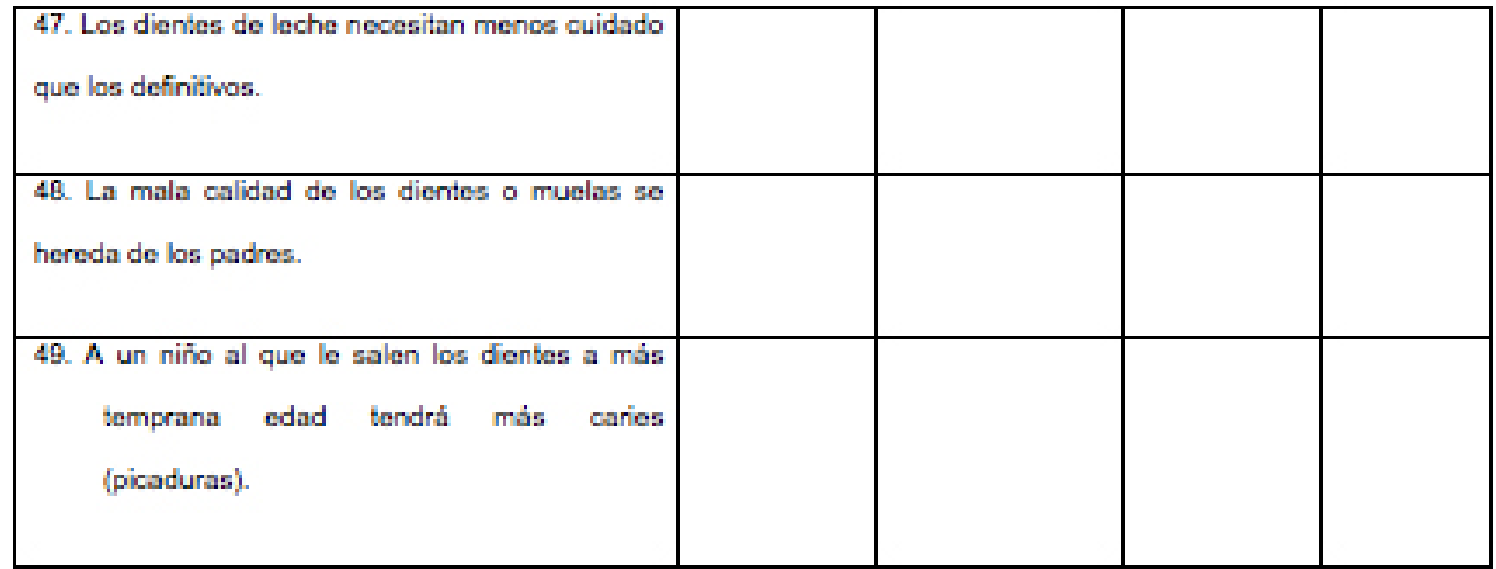

\section{Dominio IV: Auto percepción de Salud Bucal y de necesidad de tratamiento.}

Este dominio intenta valorar la auto percepción de salud bucal del cuidador, del cuidador respecto del niñolniña y las necesidades de tratamientos percibidas.

50. ¿Con cuálicuáles de las siguientes frases se siente identificadola, ouando usted decide (o tiene la intención de decidir) llevar al niñainiña al dentista? (marque todo lo que aplique).

1) Yo sólo llevo al niñolniña al dentista si elfella tiene dalor o un problema con sus dientes

2) Yo llevo al nin̄olniña al dentista regularmente porque ellella fiene problemas con sus dientes o encias

3) Yo llevo al niño/niña al dentista para revisiones (controles) regulares

4) Yo no llevo al niñolniña al dentista con tanta frecuencia como el dentista me indica

5) Yo nunca llevo al niñolniñe al dentista

6) Yo temo al dentista por lo que no levo al niño/ niña al dentista

7) Yo no llevo al niño/niña al dentista porque no me ofrecen la prestacianes que necesita

8) Yo llevo al nin̄olniña al dentisła cuando tengo posibilidades económicas

51. ¿Cuál/Cuáles de las siguientes frases describe como usted (el adulto) decide cuando visitar al dentista? (marque todo lo que aplique)

1) Yo sólo voy al dentista si yo tengo dalor o si yo tengo un problema con mis dientes o enclas

2) Yo voy a mi dentista rogulamente porque tengo problemas con mis dientes o encias

3) Yo voy a mi dentista para revisiones (controles) regulares

4) Yo no voo a mi dentista con tanta freouencia como el dentista me indica

5) Yo nunca voy al dentista 
CANDIALES, Y. ; CABELLO, R.; RODRÍGUEZ, G. ; MARRÓ, L. ; MORALES, I.; URZÚA, B. \& SÁNCHEZ, J.Validación de un cuestionario para evaluar riesgo de caries en preescolares en Santiago, Chile. Int. J. Odontostomat., 12(1):135-155, 2018.

6) Yo no voy al dentista pued tengo temar

7) Yo no voy ul dentista porque no me ofrecen las prastacianes que nocesito

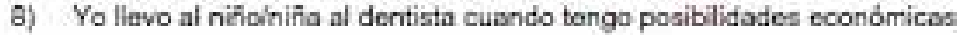

\begin{tabular}{|c|c|c|c|c|}
\hline & Muy Buena & Buena & Mala & Muy Maia \\
\hline $\begin{array}{l}\text { 52. ¿Cómo encuentra la salud derital (dientas y } \\
\text { encias) del niño'niña? }\end{array}$ & & & & \\
\hline $\begin{array}{l}\text { 53. ¿Cémo encuentra al cuidada para la salud dental } \\
\text { (dientes } y \text { anclas) del niñóniña, que usted ie } \\
\text { ontroga? }\end{array}$ & & & & \\
\hline $\begin{array}{l}\text { 54. ¿Cómo encuentra su propia salud dental (dientas } \\
\text { y ancias)? }\end{array}$ & & & & \\
\hline $\begin{array}{l}\text { 55. ¿Cómo encuentra ol cuidodo que bene can su } \\
\text { propia salud dental (dientes y encias)? }\end{array}$ & & & & \\
\hline
\end{tabular}

\section{Dominio V: Hábitos de Alimentación.}

Este daminio inzania valorar los häbitos de consumo de almentos que so encuentran vinculados con un mayor riesga individual de sufrir cories.

56. A qué edad comenzỏ al niñainiña a consumir los siguientes alimantos o bobidas? Marque con una $X$ cuando corrasponda:

\begin{tabular}{|c|c|c|c|c|c|}
\hline Rimenso & $\begin{array}{l}\text { Manas da } \\
\text { t año }\end{array}$ & $\begin{array}{l}\text { Entro } 1 \text { año } y^{2} \\
\text { años }\end{array}$ & $\begin{array}{c}\text { MSE de } 2 \\
\text { años }\end{array}$ & $\begin{array}{l}\text { Nuncatha } \\
\text { coneurnido }\end{array}$ & $\begin{array}{l}\text { No se No } \\
\text { recuardo }\end{array}$ \\
\hline Bebidas gaseocas & & & & & \\
\hline Chocolate & & & & & \\
\hline Galletas dulcos & & & & & \\
\hline
\end{tabular}




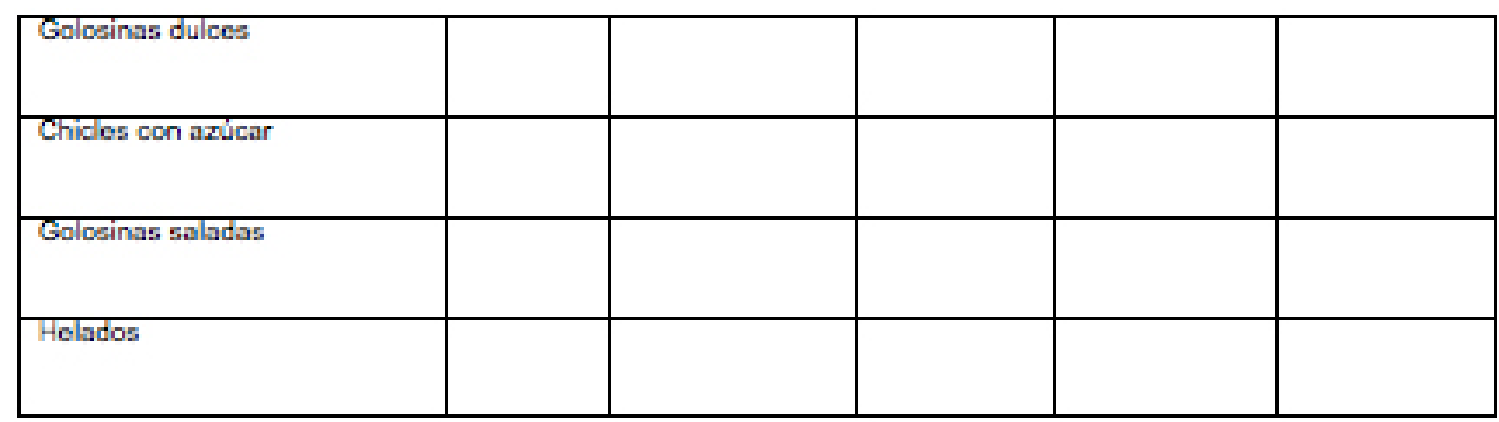

57. ¿Con qué freouencia el niño/a torna liquidos de una mamadera?

\begin{tabular}{|l|l|l|l|}
\hline Nunca___ & Casi nunca___ & Casisiampro___ & Siampro___ \\
\hline
\end{tabular}

58. ¿Toma el niña/niña generalmente liquidas de un vasito con tapa? (mostrar lámina $\mathrm{N}^{3}$ 1)

\begin{tabular}{|l|l|l|l|}
\hline Nunce___ Casi nunca___ & Casisiampre___ & Siampro___ \\
\hline
\end{tabular}

59. ¿Con qué frecuencia el niñainiña utiliza el vasito con tapa entre comidas?

\begin{tabular}{|l|l|l|l|}
\hline Nunca___ Casi nunca___ & Casisiempre___ & Simpre___ \\
& & & \\
\hline
\end{tabular}

60. ¿Usa o toma regularmente el niñofniña algún liquido, leche o jarabe a la hora de dormir o durante la noche?

\begin{tabular}{|l|l|l|l|}
\hline Nunca___ Casi nunca___ & Casisiampre___ & Siampro___ \\
& & & \\
\hline
\end{tabular}

61. ¿ El niñohniña, come alimentas entre comidas las dias de semana?

\begin{tabular}{|l|l|l|l|}
\hline Nunca___ Casi nunca___ & Casisiampre___ & Siampro___ \\
\hline
\end{tabular}

62. ¿El niñohniña, come alimentos entre comidas las dias del fin de semana? 
CANDIALES, Y. ; CABELLO, R.; RODRÍGUEZ, G. ; MARRÓ, L. ; MORALES, I.; URZÚA, B. \& SÁNCHEZ, J.Validación de un cuestionario para evaluar riesgo de caries en preescolares en Santiago, Chile. Int. J. Odontostomat., 12(1):135-155, 2018.

\begin{tabular}{|l|l|l|l|}
\hline Nunce___ Casi nunca___ & Campre & Casiempre___ & - \\
\hline
\end{tabular}

63. ¿Qué clases de alimentos come el niña/niña generalmente entre comidas regulares? Por fawor, lea la lista y marque todas las comidas que apliquen.

1) Masas salodas

2) Caramelos

3) Yogurt sin azĹcar

4) Yogurt con axúcar

5) Cereales con azúcar

Frutas frescas con azúcar o almibar

Frutos secos
8) Masas dulces
9) Azúcar
10) Choodates
11) Postres con azúcar
12) Frutas frescas sin azúcar
13) Otros

64. ¿Qué bebe generalmente el niñala niña entre comidas? Por favor, lea la lista y marque todas las bebidas que apliquen.
1) Agua de la llave
2) Agua minerdl libre de azúcar
3) Jugo natural de fruta sin azúcar
4) Liquidoe azucarados (Té, bebidas gaseosas, jugos preparados y jugos para preparar, azucarados)
5) Lácteos azucarados ( Leche, leche cultivada, yogurt liquido, endulzados con azúcar)
6) Liquidos sin axúcar (TÉ, bebidas gaseosas, jugos proparsdos y para preparar, endulzados con edulcorante)
7) Lácteos sin azúcar ( Leche, leche oultivada, yogurt liquido, endulzados con odulcorante)
a) Otros

65. ¿Con qué frecuencia toma el niño/niña, un liquido que no ses agua entre comidas durante al dia? (no cuente el desayuno, el almuerzo, la once y la cena)-

\begin{tabular}{|l|l|l|l|l|}
\hline Más de una vez a día & Una vez al dia & Agunas veces a la semana & Algunas veces al mes & Nunca \\
\hline & & & & \\
\hline
\end{tabular}

66. ¿Con qué frecuencia el niño/niña toma agua de la llave u otras bebidas preparadas con agua de la llave? 
CANDIALES, Y. ; CABELLO, R.; RODRÍGUEZ, G. ; MARRÓ, L. ; MORALES, I.; URZÚA, B. \& SÁNCHEZ, J. Validación de un cuestionario para evaluar riesgo de caries en preescolares en Santiago, Chile. Int. J. Odontostomat., 12(1):135-155, 2018.

76. ¿Con qué frecuencia ayuda usted al niño/niña a cepilarle los dientes loe dias de semana?

\begin{tabular}{|l|l|l|l|}
\hline Nunca___ Casi nunca___ & Casiempre____ & Siempre_ \\
\hline
\end{tabular}

77. ¿Con qué frecuencia ayuda usted al niñóniña a cepilarle los dientes los dias del fin de semana?

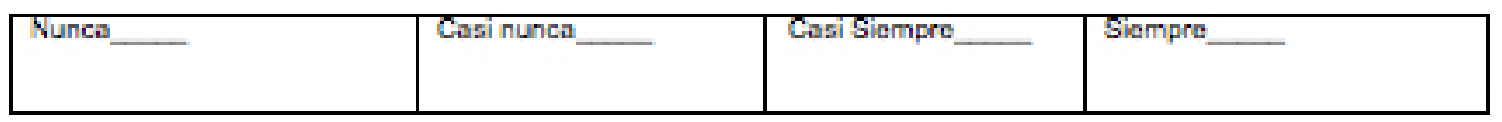

78. Cuando su niño/niña se cepilla las dientes los dias de semana: ¿Usa pasta de dientes?

\begin{tabular}{|l|l|l|l|}
\hline Nunce___ Casi nunca___ & Casisiempre___ & Simpre_ \\
& & & \\
\hline
\end{tabular}

79. Cuando su niño/niña se cepilla las dientes los dias del fin de semana: ¿Usa pasta de dientes?

\begin{tabular}{|l|l|l|l|}
\hline Nunca___ Casi nunca___ & Camprempre___ & Siempre \\
\hline
\end{tabular}

80. En las dias de semana, el niño/niña: ¿Se cepilla rogularmente las dientes después de comer, beber o consumir algủn jarabe (remedio), antes de dormir?

\begin{tabular}{|l|l|l|l|}
\hline Nunce___ Casi nunca___ & Campre_ & & \\
& & & \\
\hline
\end{tabular}

81. En las dias del fin de semana, el niño/niña ¿Se cepila regularmente los dientes después de comer, beber consumir algún jarabe (remedio), antes de dormir?

\begin{tabular}{|l|l|l|l|}
\hline Nunca___ Casi nunca___ & Casisiempre___ & Siampre___ \\
\hline
\end{tabular}


CANDIALES, Y. ; CABELLO, R.; RODRíGUEZ, G. ; MARRÓ, L. ; MORALES, I.; URZÚA, B. \& SÁNCHEZ, J.Validación de un cuestionario para evaluar riesgo de caries en preescolares en Santiago, Chile. Int. J. Odontostomat., 12(1):135-155, 2018.

92. ¿Usa el niñoiniña algún oł̣o producto para limpiar su boca? Si

No ¿Cuál?

83. ¿Cuándo comenzó el cepillado/la limpieza de las dientes del niñol niña? (marque todo lo que aplique)

1) Cuanda le salió el primer diente en la boca

2) Cuando era menor de 1 aña

3) Mayor de 1 aก๊๋ - menor de 2 años

4) Mayor de 2 años y menor de 3 años

5) Mayor de 3 aก̃กร

6) No ha comenzado el cepillada/limpieza de las dientes todavia

7) No lo recuerdo o no lo sé

84. ¿Con que freouencia usted se cepilla los dientes?

\begin{tabular}{|l|l|l|l|l|}
\hline Más de 2 veces al dia__ & 2 veoes al dia___ & Una vez al dia__ & Algunas veces en la semana__ & Nunca__ \\
\hline
\end{tabular}

85. ¿Cuándo usted se cepilla los dientes, usa pasta de dientes?

\begin{tabular}{|l|l|l|l|}
\hline Nunca___ Casi Siempre___ & Canci ___ & Campre__ \\
\hline
\end{tabular}

86. ¿Con que frecuencia usted usa seda o hilo dental en sus dientes?

\begin{tabular}{|l|l|l|l|}
\hline Más de una vez ald dla___ & Una vez al dla__ & Agunas veces a la semana_- & Nunca___ \\
\hline
\end{tabular}


CANDIALES, Y. ; CABELLO, R.; RODRÍGUEZ, G. ; MARRÓ, L. ; MORALES, I.; URZÚA, B. \& SÁNCHEZ, J. Validación de un cuestionario para evaluar riesgo de caries en preescolares en Santiago, Chile. Int. J. Odontostomat., 12(1):135-155, 2018.

\section{Dominio Vll: Exposición a microorganismos.}

Este dominio valora la exposición a microorganiemos, dal biôlon bucal a loe que el niño está expuesto, como principal fuente el cuidador.

87. ¿Con qué freouencia limpia el chupete (Tete) de su niñolniña con su boca antes de dárselo a élvella?

\begin{tabular}{|l|l|l|l|l|}
\hline Nunce____ Casi nunca___ & Casi Siempre__ & Siempre___ & No _ — \\
& & & & \\
\hline
\end{tabular}

8B. ¿Con qué frecuencia besa al nin̄olniñe en la boca?

\begin{tabular}{|l|l|l|l|}
\hline Nunca___asi nunca___ & Casiompre & & \\
\hline
\end{tabular}

89. ¿Con qué freouencia prueba usted las comidas/bebidas del niño/niña usando la misma cuchara, tenedor, vaso, u atro?

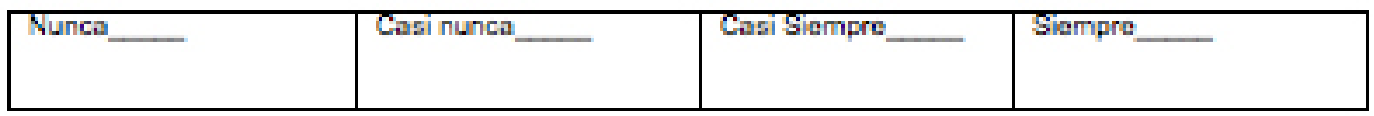

90. ¿Con quá frocuencia limpia el helado, dulce, fruta u atro alimento de su niñainiña con su boca antes de dárselo élvella?

\begin{tabular}{|l|l|l|l|}
\hline Nunca__asi nunca___ & Casisiempre___ & Siempre___ \\
\hline
\end{tabular}

91. En la casa: ¿Comparte el niñolniña el cepillo de dientes con alguien?

92. En el Jardin: ¿Comparte el niñofniña el cepillo de dientes con alguien?

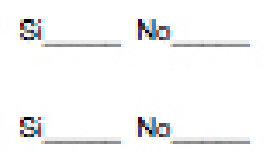

Si__ No____

\section{Identíficación y Contacto}

93. Nombres del niñalniña: Apellidos:

94. Sexo del niñainiña:

95. Fecha de Nacimiento del nin̄olniña: กiกัa:

niña:

dia: Imes: /aña: 
CANDIALES, Y. ; CABELLO, R.; RODRÍGUEZ, G. ; MARRÓ, L. ; MORALES, I.; URZÚA, B. \& SÁNCHEZ, J. Validación de un cuestionario para evaluar riesgo de caries en preescolares en Santiago, Chile. Int. J. Odontostomat., 12(1):135-155, 2018.

96. Nombres del encuestado:

Apellidos:

97. Fecha de nacimiento del encuestadoc diax imes: /aña:

98. Su relación con el niño/niña [madre/padre, abuela/abuela, tíatio, apoderado, padre adoptivo, otro):

99. ¿Quién, además de usted, cuida regulamente al niño/niña?

100. ¿Cuál es su direcoión actual?

Calle No Depto. Comuna Ciudad

Urbano Rural (No llenar)

101. ¿Cuál es su nủmero de teléfono actual?

102. ¿Hay otro número en el cuál nosobros podamos localzarlo?

103. ¿Tiene correo electrónico?, ¿Cuál es?

104. Si nosatros tenemce problemas localizándalo(a) en el futuro: ¿Quién sabría donde localizarla(a) en el curso del próximo aña?

a. Nombre: b. Relación con usted:

c. Número de teléfono: c. Conreo electrónico:

d. Dirección:

Calle Nopta. Comuna Ciudad

105. Tipo de hogar. (Consultar sobre composición y clasificar)

Unipersonal: (una sola persona que es el (la) jefe/a de hagar)

Nuclear Biparental: (matrimanio o unión de hecho con hijoe o hijastros)

Nuclear Monaparental: (jefola de hagar con hijos o hijastros)

Extenso Biparental: (hogar nuclear biparental más cualquier otro pariente del jefela de hagar no nuclear)

Extenso Monoparental: (hogar nuclesr monoparental más cualquier obro pariente dal jefo/a de hogar no nudear)

Compuesto Biparental: (hogar nuclear biparental o un hogar extenso biparental más no parientes)

Compuesto Monoparontal: (hogar nuclear monoparental o un hogar extenso monoparental más no parientes)

Hogar sin núcleo: (hogar en que no está presente el núcleo familiar primario)

106. ¿Cuántos adultos de los que viven en la casa donde vive el niñolniña, tienen trabajo remunerado? 
CANDIALES, Y. ; CABELLO, R.; RODRÍGUEZ, G. ; MARRÓ, L. ; MORALES, I.; URZÚA, B. \& SÁNCHEZ, J. Validación de un cuestionario para evaluar riesgo de caries en preescolares en Santiago, Chile. Int. J. Odontostomat., 12(1):135-155, 2018.

107. Considerando a todas las personas que viven con el niñoiniña y aportan dinero on al hogar: ¿En qué rango de ingresos mensuales se ubicaria su hogar?

1) Menas de $\$ 80.000$

2) Entre $\$ 81.000$ y $\$ 150.000$ al mes

3) Entre $\$ 151.000$ y $\$ 220.000$ al mes

4) Entre $\$ 221.000$ y $\$ 280.000$ al mes
5) Entre $\$ 281.000$ y $\$ 450.000$ al mes

6) Entre $\$ 451.000$ y $\$ 780.000$ al mes

7) Más de $\$ 780.000$ al mes

100. Considerando a todas las personas que forman parte del hogar nuelear del niñolniña y que aportan dinero: ¿En qué rango de ingresos mensuales se ubicaria su hogar?

1) Menos de $\$ 80.000$

2) Entre $\$ 81.000$ y $\$ 150.000 \mathrm{al}$ mes

3) Entre $\$ 151.000$ y $\$ 220.000 \mathrm{~d}$ mes

4) Entre $\$ 221.000$ y $\$ 200.000$ al mes

Pregunta Global final:

1. Frente a la afirmación "Mi niño o niña se encuentra en riesgo de suffri Caries" ustod se enouentra: Marque con uns $\mathrm{X}$

\begin{tabular}{|l|l|l|l|}
\hline Muy desacuerdo ___ & De acuerdo___ & Desacuerdo___ & Muy desacuerdo___ \\
\hline
\end{tabular}

Los criterios de inclusión fueron: sanos sistémicamente, y que asistan a un jardín infantil privado o de la Fundación Integra en la Región Metropolitana de Santiago de Chile.

Los criterios de exclusión fueron: niños con alguna discapacidad motora, hijos de padres extranjeros y aquellos cuidadores o representantes legales que no quisieron firmar el consentimiento informado.

El grupo de alto riesgo de caries fue definido como niños que asisten a jardines infantiles de bajo nivel socioeconómico, perteneciente a la FUNDACIÓN INTEGRA, y que poseían una o más lesiones de caries cavitadas; y el grupo de bajo riesgo fue definido como niños que asisten a jardines infantiles de dependencia particular, de alto nivel socioeconómico y que no poseían lesiones de caries cavitadas.

\author{
5) Entre $\$ 281.000$ y $\$ 450.000$ al mes \\ 6) Entre $\$ 451.000$ y $\$ 780.000$ al mes \\ 7) Más de $\$ 780.000$ al mes
}

La caracterización socioeconómica nacional se realizó según la encuesta CASEN (CASEN, 2009).

Recolección de datos. El equipo de trabajo estuvo formado por: 2 Odontólogos clínicos con experiencia, capacitados y previamente calibrados en el sistema ICDAS II; 2 Registradores; 2 coordinadores de trabajo de campo por establecimiento y 2 encuestadores.

La evaluación se realizó en las salas de los centros educacionales con luz artificial LED frontal (Atom ${ }^{\circledR}$ ), una por cada odontólogo clínico. Se contó con ficha de registro de examen clínico y formulario de datos de identificación. Para el examen clínico se utilizó: espejo plano; sonda CPITN con extremo en forma de bolita de $0,5 \mathrm{~mm}$; cotonitos de algodón para secar superficie dentaria; guantes y mascarilla desechables. 
Análisis estadístico. Para el análisis de validez de discriminación, la variable puntaje se describió con medidas de tendencia central y dispersión. Se evaluó la asociación entre el puntaje del cuestionario y la pertenencia al grupo de alto y bajo riesgo a través de un modelo de regresión logística, con el reporte del odds ratio (OR) y su respectivo intervalo del $95 \%$ de confianza, y posteriormente se confeccionó una curva ROC (Receiver Operating Characteristic) estimándose el área bajo la curva para establecer la capacidad de predicción. Se evaluó de la misma manera la asociación entre la pregunta global y el puntaje. Para el análisis de la consistencia interna se usó el Cálculo de alpha de Conbrach. Para el cálculo de la estabilidad temporal del cuestionario se usó el índice de Kappa de Cohen para las variables categóricas y el índice de concordancia intraclase para las variables cuantitativas. Se utilizó el programa Stata 12.1 para el análisis.

\section{RESULTADOS}

Los resultados obtenidos relacionados a la historia de caries arrojó un índice ceo-d de 3,89.

Validez de discriminación. Según el análisis estadístico de modelo de regresión logística se obtiene que sí existe asociación estadísticamente significativa entre el puntaje del cuestionario y el grupo riesgo de caries (bajo y alto): $p=0,001$ odss ratio (OD) 1,$961 ; 95 \%$ intervalo de confianza $(\mathrm{Cl}) 1,375-2,795$.

Las medidas de tendencia central y dispersión del puntaje del cuestionario versus el grupo de bajo y alto riesgo de caries, se muestran en la Figura 1.

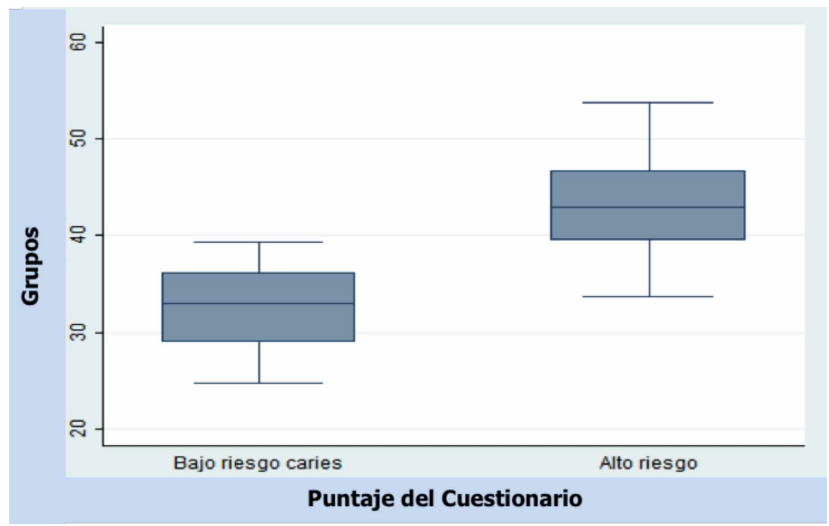

Fig. 1. Medidas de tendencia central y dispersión del puntaje del cuestionario versus el grupo de bajo y alto riesgo de caries.
En la Figura 2 se muestra una curva de rendimiento diagnóstico o curva ROC, con un área bajo la curva de 0,95; es decir, con un alto poder de discriminación para individuos con alto o bajo riesgo de caries.

Validez de Convergencia. Según el análisis estadístico de modelo de regresión logística se obtiene que no existe asociación estadísticamente significativa entre el puntaje del cuestionario y la pregunta global dicotomizada: $p=0,193$; odss ratio (OD) 1,$061 ; 95 \%$ intervalo de confianza $(\mathrm{Cl}) 0,970-1,161$.

Las medidas de tendencia central y dispersión del puntaje del cuestionario versus la pregunta global victimizada, se observan en la Figura 3.

En la Figura 4 muestra una curva de rendimiento diagnóstico o curva ROC con un área bajo la curva de 0,61 ; es decir, con un aceptable poder de discriminación para el puntaje de cuestionario y la pregunta global dicotomizada.

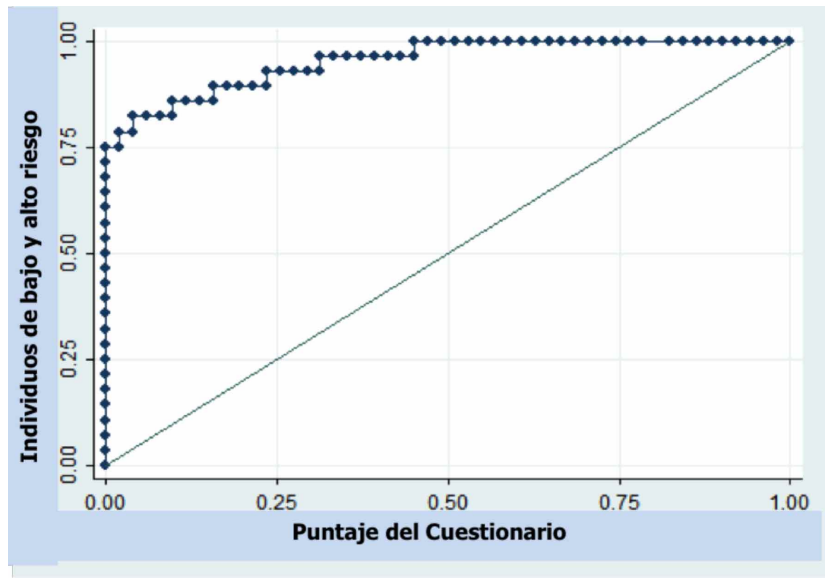

Fig. 2. Curva de ROC muestra un área bajo la curva 0,95 entre el puntaje del Cuestionario y los individuos de bajo y alto riesgo.

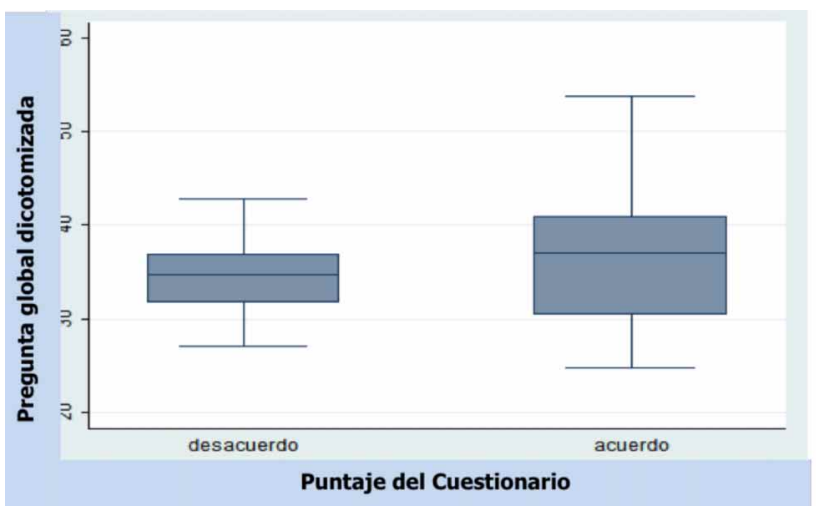

Fig. 3. Medidas de tendencia central y dispersión del puntaje del cuestionario versus la pregunta global dicotomizada. 
Consistencia Interna. Se obtuvo un valor de 0,72 considerado como aceptable.

Estabilidad Temporal. El índice obtenido osciló entre 0,37 a 1 ; es decir, un fiabilidad de buena a muy buena.

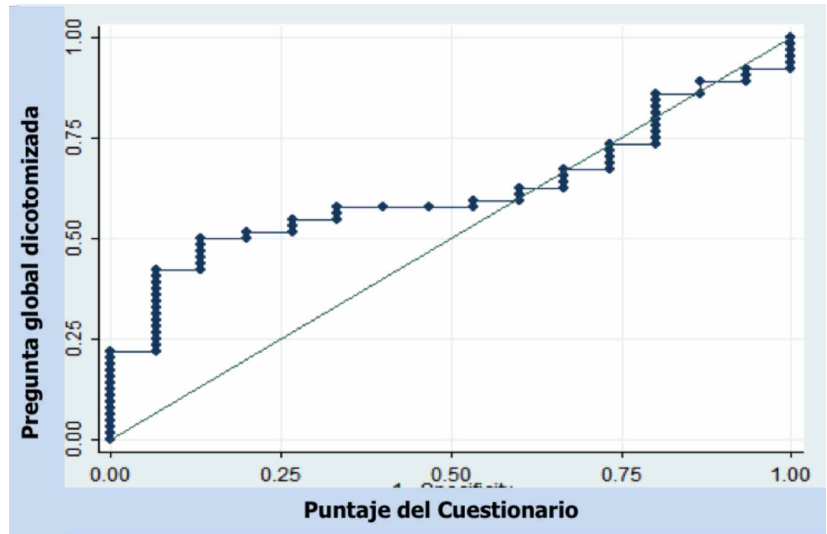

Fig. 4. Curva de ROC muestra un área bajo la curva 0,61 entre el puntaje del Cuestionario y la pregunta global dicotomizada.

\section{DISCUSIÓN}

El presente estudio validó un cuestionario para evaluar riesgo de caries en preescolares. La validez de discriminación del presente cuestionario mostró una curva de rendimiento diagnóstico o curva ROC, con un área bajo la curva de 0,95 , es decir, con un alto poder de discriminación para individuos con alto o bajo riesgo de caries, por lo tanto, confirma la hipótesis planteada que el presente cuestionario si es un instrumento válido para identificar factores de riesgo asociados al desarrollo de caries en preescolares de 24 a 71 meses de edad de la Región Metropolitana.

El estudio de la estabilidad temporal mediante comparaciones test - retest calculado con el índice de Kappa de Cohen osciló entre 0,37 a 1, en la mayoría de los dominios mostrando una fiabilidad de buena a muy buena, a excepción de las preguntas 44 y 47 del dominio III constructo social de la enfermedad, la cual mostró una fiabilidad débil, esto puede deberse a que los cuidadores principales no tienen un criterio muy establecido del contexto social de la enfermedad de caries.

Estudios previos que utilizaban cuestionarios que solo consideraban algunos factores de forma aislada: examen dental, análisis de saliva, estudios microbiológicos, pH de la placa bacteriana. Estos cuestionarios no han sido validados previo a su aplicación en la muestra de estudio, lo que hace difícil la comparación de resultados y la reproducción de los mismos (Ramos-Gómez et al.; Kalwitzki et al.; Marshall et al.; Finlayson et al.; Patir et al.; Gao et al.; MacRitchie et al.).

En el estudio de MacRitchie et al., el cual aplicó un cuestionario en un modelo biopsicosocial que incluyó un examen oral, pruebas salivales, microbiológicas y de $\mathrm{pH}$ de la placa bacteriana, en donde se obtuvo una sensibilidad de $82 \%$ (MacRitchie et al.).

Los resultados obtenidos en nuestro estudio relacionados a la historia de caries arrojó un índice ceo-d de 3,89 que permite comparar con otras investigaciones epidemiológicas reportadas en la literatura que han utilizado el criterio estándar de la OMS para la detección de caries. En Chile, en el año 2007, Ceballos et al. publicaron un estudio donde se estimó la prevalencia de caries en niños de 2 a 4 años (ceo-d: 0,54 y 2,32 respectivamente) de la Región Metropolitana, mientras que Soto et al. estimaron la prevalencia de caries en niños de 6 años (ceod: 3,71 ) a nivel nacional.

El presente cuestionario es una versión original, diseñado por un grupo de profesionales considerados expertos en Cariología, Odontología Restauradora y Metodología de la investigación y Salud Pública, en su construcción se prestó especial atención a los contenidos y el contexto de cada pregunta. Las versiones iniciales fueron revisadas por el comité revisor integrado por nuestro equipo investigador, que corrigió detalladamente el lenguaje y su contenido. La ponderación para el análisis de las diferentes variables, fue asignada por expertos seleccionados considerando su experiencia profesional e investigativa en el ejercicio de la odontología. Durante el ejercicio de definir la puntuación del cuestionario, la ponderación asignada a los ítems y escalas de medición de las diferentes dimensiones, se realizó de acuerdo a las apreciaciones y pericia de los integrantes del equipo investigador. Se determinó según importancia el peso relativo que cada pregunta (o dimensión) tendría sobre el puntaje total del cuestionario.

El proceso sistemático de diseño por expertos desarrollado durante nuestro estudio, ha permitido satisfacer la necesidad de contar con un instrumento validado que sea capaz de recolectar y analizar los dife- 
rentes factores de riesgo de caries dental a nivel de las comunidades preescolares más vulnerables y poder así obtener información confiable para diseñar y planificar estrategias de intervención en el control y prevención de la caries dental en la comunidad de preescolares a nivel nacional.

La prueba piloto permitió identificar la dificultad de comprensión lingüística de las preguntas de cada dominio del cuestionario. Las recomendaciones sugeridas durante las entrevistas grupales a un subgrupo de cuidadores que participaron en este piloto y a un subgrupo de los encuestadores que aplicaron el cuestionario, fueron analizadas cuidadosamente. Esto permitió optimizar cada una de las etapas de validación del instrumento y estimar el tiempo para contestar el cuestionario que fue de 30 a $40 \mathrm{~min}$.

Para determinar la validez de convergencia se comparó el resultado del cuestionario contra una afirmación que permitió evaluar la creencia del individuo respecto de la condición de riesgo mediante una pregunta global (PG), al dicotomizar esta variable en sólo dos grupos : bajo riesgo y alto riesgo, se pierde información dejando fuera la posibilidad de categorizar a otro grupo como de moderado riesgo. Sin embargo, la posibilidad de realizar el análisis estadístico se vuelve más fácil y menos engorrosa.

Se sugiere en futuras investigaciones, la determinación de un número mayor de categorías que permitan al instrumento discriminar de manera más fina el límite entre bajo, moderado y alto riesgo. La validación se vería enriquecida al contar con un número mayor de categorías que entregara a su vez, una mayor cantidad de datos para el análisis.

La consistencia interna obtenida del instrumento fue de 0,72 . El alfa establece que debiera ser igual o superior a 0,7 para obtener una confiabilidad estándar en investigación. Sugiere que la escala del cuestionario presenta una homogeneidad aceptable que otorga confiabilidad al instrumento. El cálculo comprendió la correlación escala-escala para cada dimensión del cuestionario, la confiabilidad mejora en la medida que el número de observaciones aumenta por tanto, a mayor número de preguntas presentes en un instrumento de medición, mayor será la confiabilidad, el presente estudio comprendió un cuestionario con un total de 108 preguntas observadas y analizadas, que aumentó su confiabilidad.

En relación a la estabilidad temporal del cuestio- nario, que mostró en la mayoría de los dominios una fiabilidad de buena a muy buena, siendo favorecida por el tiempo transcurrido entre la 1ra aplicación y la 2da. aplicación que fue de 7 - 10 días, lo que garantiza la fiabilidad de las respuestas.

\section{CONCLUSIONES}

El presente cuestionario es un instrumento válido para discriminar riesgo de caries, permitiendo un mejor análisis de los determinantes de la caries dental en la población preescolar chilena.

CANDIALES, Y. ; CABELLO, R.; RODRÍGUEZ, G. ; MARRÓ, L. ; MORALES, I.; URZÚA, B. \& SÁNCHEZ, J. Validation of a questionnaire to assess risk of caries in preschool-age children in Santiago, Chile. Int. J. Odontostomat., 12(1):135-155, 2018.

ABSTRACT: Early Childhood Caries (ECC) is an aggressive form of tooth decay, and the last national unpublished reports reveal a caries prevalence of $50 \%$ at 4 years of age in children, in the Santiago Metropolitan Region (Soto et al., 2007). The objective of this study is to validate a questionnaire that allows the collection of information related to several caries risk factors in preschoolchildren. For the validation of the questionnaire, the convergence and discrimination validity, the internal consistency and the retest, test reliability of the instrument were determined in two independent samples. The instrument was applied to the main caregiver of 118 preschoolers between 24 and 71 months of age, who attend private childcare centers (low caries risk) and the INTEGRA Foundation childcare (high caries risk) in the Metropolitan Region of Santiago, Chile. Dental clinical examinations were performed by two calibrated dentists using the OMS and ICDAS II criteria. A logistic regression model was estimated and the ability to discriminate the score through an ROC curve was evaluated. The questionnaire showed a validity of discrimination of 0.95 between both groups and of the global question 0.61 and an internal consistency of the questionnaire of 0.72 . In the convergence validity, it was found that there is no statistically significant association between the questionnaire score and the dichotomized global question (RE) 1.061. However, it was evidenced that there is a statistically significant association between the questionnaire score and the caries risk group (low and high) (OD) 1.961. Temporal stability by means of Test - Retest comparisons calculated with the Kappa index ranged from 0.37 to 1 . Therefore, it can be concluded that the present questionnaire is a valid instrument for discriminating caries risk, allowing a better analysis of the determinants of dental caries in the Chilean preschool population.

KEY WORDS: dental caries, risk assessment, preschool-aged children. 


\section{REFERENCIAS BIBLIOGRÁFICAS}

Baelum, V.; van Palenstein Helderman, W.; Hugoson, A.; Yee, R. \& Fejerkov, O. A global perspective on changes in the burden of caries and periodontitis: implications for dentistry. J. Oral Rehabil., 34(12):872-906, 2007.

CASEN 2009. Encuesta de Caracterización Socioeconómica Nacional. Santiago de Chile, Gobierno de Chile, 2009. Ministerio de Planificación, Disponible en: http://www.superacionpobreza.cl/wpcontent/uploads/2014/01/resultados_casen_2009.pdf

Ceballos, M.; Acevedo, C.; Corsini, G. \& Jans, A. Diagnóstico de Salud Bucal de Niños de 2 y 4 años, que Asisten a la Educación Preescolar en la Región Metropolitana. Santiago de Chile, Gobierno de Chile, Ministerio de Salud, 2007.

Edelstein, B. L. The dental caries pandemicand disparities problem. BMC Oral Health, 6(Suppl. 1):S2, 2006.

Finlayson, T. L.; Siefert, K.; Ismail, A. I. \& Sohn, W. Psychosocial factors and early childhood caries among low-income AfricanAmerican children in Detroit. Community Dent. Oral Epidemiol., 35(6):439-48, 2007.

Gao, X. L.; Hsu, C. Y.; Xu, Y.; Hwarng, H. B.; Loh, T. \& Koh, D. Building caries risk assessment models for children. J. Dent. Res., 89(6):637-43, 2010.

Kalwitzki, M.; Weiger, R.; Axmann-Krcmar, D. \& Rosendahl, R. Caries risk analysis: considering caries as an individual timedependent process. Int. J. Paediatr. Dent., 12(2):132-42, 2002.

MacRitchie, H. M.; Longbottom, C.; Robertson, M.; Nugent, Z., Chan, K.; Radford, J. R. \& Pitts, N. B. Development of the Dundee Caries Risk Assessment Model (DCRAM)--risk model development using a novel application of CHAID analysis. Community Dent. Oral Epidemiol., 40(1):37-45, 2012.

Marshall, T. A.; Levy, S. M.; Broffitt, B.; Warren, J. J.; EichenbergerGilmore, J. M.; Burns, T. L. \& Stumbo, P. J. Dental caries and beverage consumption in young children. Pediatrics, 112(3 Pt. 1):e184-91, 2003.

Patir, A.; Seymen, F.; Yildirim, M.; Deeley, K.; Cooper, M. E.; Marazita, M. L. \& Vieira, A. R. Enamel formation genes are associated with high caries experience in Turkish children. Caries Res., 42(5):394400, 2008.

Petersen, P. E. The World Oral Health Report 2003: continuous improvement of oral health in the 21st century--the approach of the WHO Global Oral Health Programme. Community Dent. Oral Epidemiol., 31 Suppl. 1:3-23, 2003.

Ramos-Gómez, F.; Jue, B. \& Bonta, C. Y. Implementing an infant oral care program. J. Calif. Dent. Assoc., 30(10):752-61, 2002.

Soto, L.; Tapia, R. \& Jara, G. Diagnóstico Nacional de Salud Bucal del Adolescente de 12 Años y Evaluación del Grado de Cumplimiento de los Objetivos Sanitarios de Salud Bucal 2000- 2010. Santiago de Chile, Ediciones de Universidad Mayor, 2007.
Corresponding author: Jenny Sánchez González Área de Cariología Departamento de Odontología restauradora Facultad de Odontología Universidad de Chile Sergio Roberto Livingstone Polhammer 943 Independencia Región Metropolitana CHILE

Email: jsanchez@odontologia.uchile.cl

Recibido : 07-11-2017

Aceptado: 06-01-2018 